\title{
Eastward-moving convection-enhanced modons in shallow water in the equatorial tangent plane $\odot$
}

Cite as: Phys. Fluids 31, 021701 (2019); https://doi.org/10.1063/1.5080415

Submitted: 08 November 2018 . Accepted: 15 January 2019 . Published Online: 04 February 2019

M. Rostami, and V. Zeitlin

\section{COLLECTIONS}

F This paper was selected as Featured
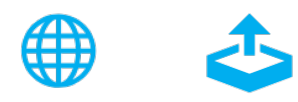

\section{PHYSICS TODAY}

WHITEPAPERS
ADVANCED LIGHT CURE ADHESIVES

Take a closer look at what these

environmentally friendly adhesive

systems can do
READ NOW

PRESENTED BY 8 MASTERBOND: 


\title{
Eastward-Moving Convection-Enhanced Modons in Shallow Water in the Equatorial Tangent Plane
}

\author{
MASOUD ROSTAMI $^{a, b}, \operatorname{VLADIMIR}_{\text {ZEITLIN }}{ }^{a}$ \\ ${ }^{a}$ Laboratoire de Météorologie Dynamique (LMD)/IPSL, Sorbonne Universités and Ecole \\ Normale Supérieure(ENS), Paris, France \\ ${ }^{b}$ Institute for Geophysics and Meteorology (IGM), University of Cologne, Cologne, Ger- \\ many
}

This is the Authors Original Manuscript (AOM); that is, the manuscript in its original form; a preprint . The Version of Record of this manuscript has been published and is available in the: "AIP Publishing - Physics of Fluid": https://doi.org/10.1063/1.5080415 


\title{
Eastward-Moving Convection-Enhanced Modons in Shallow Water in the Equatorial Tangent Plane
}

\author{
M. Rostami ${ }^{1,2}$, V. Zeitlin ${ }^{1, *}$ \\ ${ }^{1}$ LMD, Sorbonne University and Ecole Normale Supérieure, \\ 24 rue Lhomond, 75005 Paris, France \\ ${ }^{2}$ Institute for Geophysics and Meteorology (IGM), \\ University of Cologne, Cologne, Germany
}

\begin{abstract}
We report a discovery of steady long-living slowly eastward moving large-scale coherent twin cyclones, the equatorial modons, in the shallow water model in the equatorial beta-plane, the archetype model of the ocean and atmosphere dynamics in tropics. We start by constructing analytical asymptotic modon solutions in the non-divergent velocity approximation, and then show by simulations with a high-resolution numerical scheme that such configurations evolve into steady dipolar solutions of the full model. In the atmospheric context, the modons persist in the presence of moist convection, being accompanied and enhanced by specific patterns of water-vapour condensation.
\end{abstract}

\footnotetext{
* Corresponding author. Email: zeitlin@lmd.ens.fr
} 
As is known, the large-scale atmosphere and ocean dynamics in tropics is largely determined by equatorial waves. Indeed, superposition of atmospheric Outgoing Longwave Radiation (OLR) data onto the spectrum of equatorial waves gives a very good correspondence [1], except for a branch corresponding to slow eastward-moving motions associated with the Madden-Julian Oscillation (MJO) [2]. The Kelvin waves, the only species of the equatorial waves that can produce steady large-scale eastward-moving structures, have much faster velocity than MJO [3]. This fact motivates a search for coherent dynamical structures with slow eastward propagation which, thus, should be necessarily nonlinear. There do exist steady eastward-moving structures in a fluid layer on the mid-latitude $f$-plane, the modons which are exact dipolar solutions of the quasi-geostrophic (QG) equations [4]. Although the classical modons were found analytically in the QG approximation, it was shown first by a computer-assisted analysis [5] and then by direct numerical simulations [6] that corresponding solutions exist also in the parent rotating shallow water (RSW) model on the $f$-plane. The QG modon solutions were extended to the full sphere $[7,8]$ in the framework of equivalent barotropic model, which is structurally close to QG. The pioneering paper [9] advanced an idea that the MJO could be related to such a modon in spherical geometry and showed that, like in the midlatitude tangent plane [6], the equivalent barotropic modon persists in the RSW on the sphere. Yet, the archetype model for understanding dynamics in the tropics is RSW in the equatorial beta-plane, e.g. [10]. We, thus, look for eastward-moving modons in this model. However, it encounters an obstacle from the very beginning, because there is no consistent $\mathrm{QG}$ approximation at the equator, e.g. [11], so the known modon solutions can not be borrowed. Our main observation is that there is a dynamical regime in RSW, called long-wave approximation in oceanography [12], which corresponds to small pressure variations, and gives, to the leading order, equations which do allow for modon solutions. In the atmospheric context this regime is the RSW analog of the non-divergent equatorial balance model [13]. The relevance of this model to large-scale tropical motions in the atmosphere is supported by scale and data analyses $[14,15]$. We construct the modon solutions in this regime and, following [6], use these asymptotic solutions to initialize high-resolution numerical simulations with the full RSW model, and show that coherent dipolar steady eastward-moving structures do arise and persist. We then show that, in the atmospheric context, inclusion of moisture, with condensation and evaporation, enhances the modons, without disrupting long-time coherence, and produces specific convective patterns. 
We start with RSW equations in the equatorial beta- plane with no dissipation:

$$
\left\{\begin{array}{l}
\partial_{t} \mathbf{v}+\mathbf{v} \cdot \nabla \mathbf{v}+\beta y \hat{\mathbf{z}} \wedge \mathbf{v}+g \nabla h=0 \\
\partial_{t} h+\nabla \cdot(\mathbf{v} h)=0
\end{array}\right.
$$

where $\nabla=\left(\partial_{x}, \partial_{y}\right), \mathbf{v}=(u, v), u$ is zonal and $v$-meridional components of velocity, $h$ is geopotential height (thickness), $\beta$ is the meridional gradient of the Coriolis parameter, and $\hat{\mathbf{z}}$ is a unit vertical vector. The interpretation of the model in the oceanic context is direct, while in the atmospheric context it should be understood as vertically averaged primitive equations in pseudo-height pressure coordinates [16], and can be extended to include water vapor with condensation and related latent heat release [17], as well as surface evaporation (see below). We introduce a pressure perturbation parameter $\lambda: h=H(1+\lambda \eta)$, where $H$ is unperturbed thickness, and fix spatial, velocity and time- scales: $(x, y) \sim L,(u, v) \sim V, t \sim L / V$. Under hypothesis that $\lambda \rightarrow 0$, and $g H \lambda / V^{2}=\mathcal{O}(1)$, hence $V<<\sqrt{g H}$, i.e. the characteristic velocity is much smaller than the phase velocity of the Kelvin waves $c=\sqrt{g H}$, the nondimensional equations take the form:

$$
\begin{gathered}
\partial_{t} \mathbf{v}+\mathbf{v} \cdot \nabla \mathbf{v}+\bar{\beta} y \hat{\mathbf{z}} \wedge \mathbf{v}+\nabla \eta=0 \\
\lambda\left(\partial_{t} \eta+\mathbf{v} \cdot \nabla \eta\right)+(1+\lambda \eta) \nabla \cdot \mathbf{v}=0
\end{gathered}
$$

where $\bar{\beta}=\beta L^{2} / V$, and $\mathbf{v}=\mathbf{v}_{0}+\lambda \mathbf{v}_{1}+\ldots$ In the leading order in $\lambda(3)$ gives $\nabla \cdot \mathbf{v}_{0}=0$, the motion is non-divergent, and $u_{0}=-\partial_{y} \psi, v_{0}=\partial_{x} \psi$. Cross-differentiation of the zonal and meridional momentum equations results in the equation for the stream-function:

$$
\nabla^{2} \psi_{t}+\mathcal{J}\left(\psi, \nabla^{2} \psi\right)+\bar{\beta} \psi_{x}=0
$$

where $\mathcal{J}$ denotes the Jacobian. The modon solutions are built following [4]. They are are obtained under hypothesis of steady motion with constant zonal velocity $U$, by supposing a linear relationship between the absolute vorticity and stream-function in co-moving frame, which gives inhomogeneous Helmholtz equation. It is solved by separation of variables in polar coordinates in terms of Bessel functions, first in the outer domain under condition of decay, and then in the inner domain, and matching the inner and outer solutions across a circle of a given radius $a$ in the plane. The solution has the form:

$$
\left\{\begin{array}{l}
\psi_{\text {ext }}=-\frac{U a}{K_{1}(p a)} K_{1}(p r) \sin \theta, \quad r>a, \\
\psi_{\text {int }}=\left[\frac{U p^{2}}{k^{2} J_{1}(k a)} J_{1}(k r)-\frac{r}{k^{2}}\left(1+U+U k^{2}\right)\right] \sin \theta, \quad r<a,
\end{array}\right.
$$



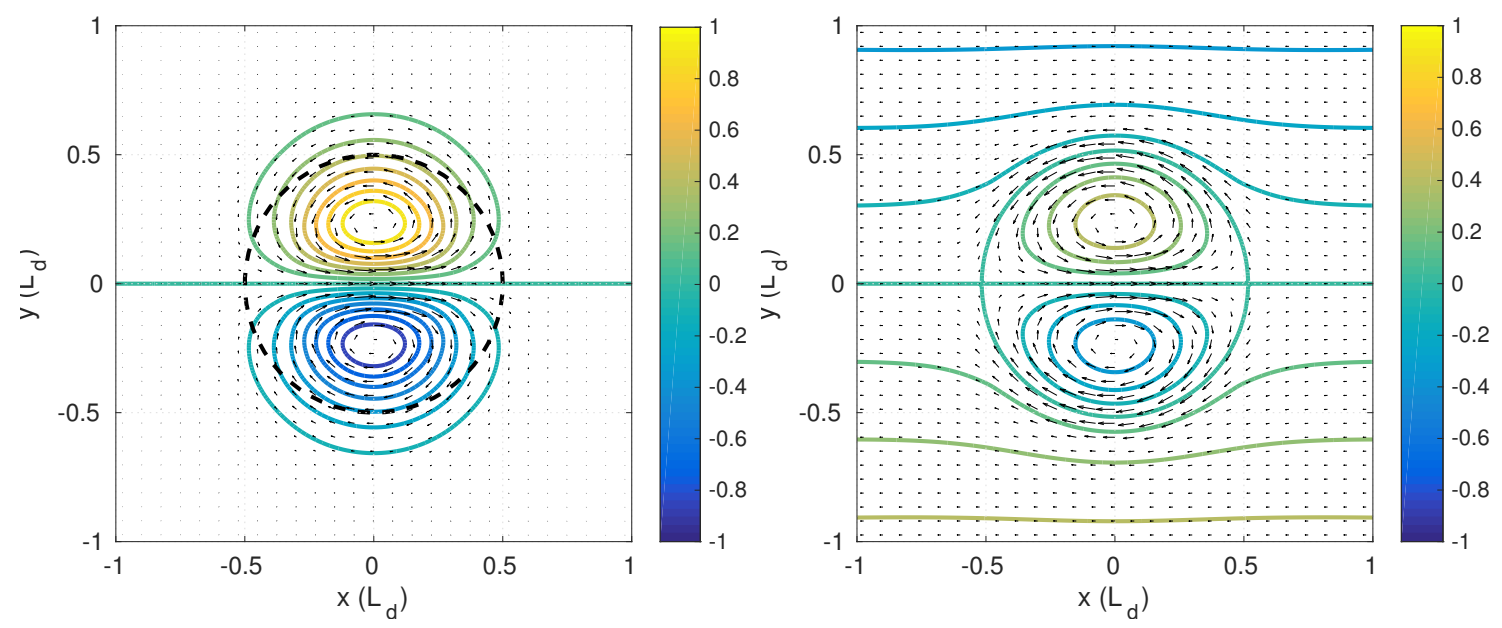

FIG. 1. Normalised streamlines and velocity field of an asymptotic modon in stationary (left ) and co-moving (right) frames. Dashed circle: separatrix of radius $a$.

where $J_{1}$ and $K_{1}$ are ordinary and modified Bessel functions of order one, respectively, $p$ is real, $p^{2}=\bar{\beta} / U$, so $U>0$, and the motion is eastward. For each pair $(a, p)$ there exist a series of eigenvalues $k$ arising from matching conditions, of which the lowest corresponds to a dipole, to be called the asymptotic modon. The next eigenvalue gives a quadrupolar solution called "shielded modon", and so on. By construction, this solution can be centred anywhere in the equatorial beta-plane, yet it is only when its center is at the equator that it results in a cyclonic pair. The streamlines of the asymptotic modon are presented in Fig. 1. First-order corrections $u_{1}, v_{1}$ can be found in the next order of the asymptotic expansion. There is no guarantee that such expansion converges, but numerical results presented below suggest that there is indeed an exact solution of (1) corresponding to this asymptotic one.

We initialized the numerical simulations in the RSW model with the velocity field corresponding to the asymptotic modon solution (5) with $U \ll \sqrt{g H}$, and flat pressure. Simulations were performed with high-resolution well-balanced finite-volume numerical scheme [18] in a rectangular domain with sponges at the boundaries, which allow to mostly evacuate short inertia-gravity waves. No explicit dissipation was added. The domain was chosen to be symmetric with respect to the equator and wide enough $9 L_{d} \times 6.5 L_{d}$, where $L_{d}=(g H)^{1 / 4} / \beta^{1 / 2}$ is the equatorial deformation radius, in order to minimize the influence of boundaries onto the modons. We varied spatial resolution from $600 \times 600$ to $1200 \times 1200$, to check numerical convergence. The natural units of length and time in the numerical scheme are $L_{d}$ and $1 / \beta L_{d}$, 


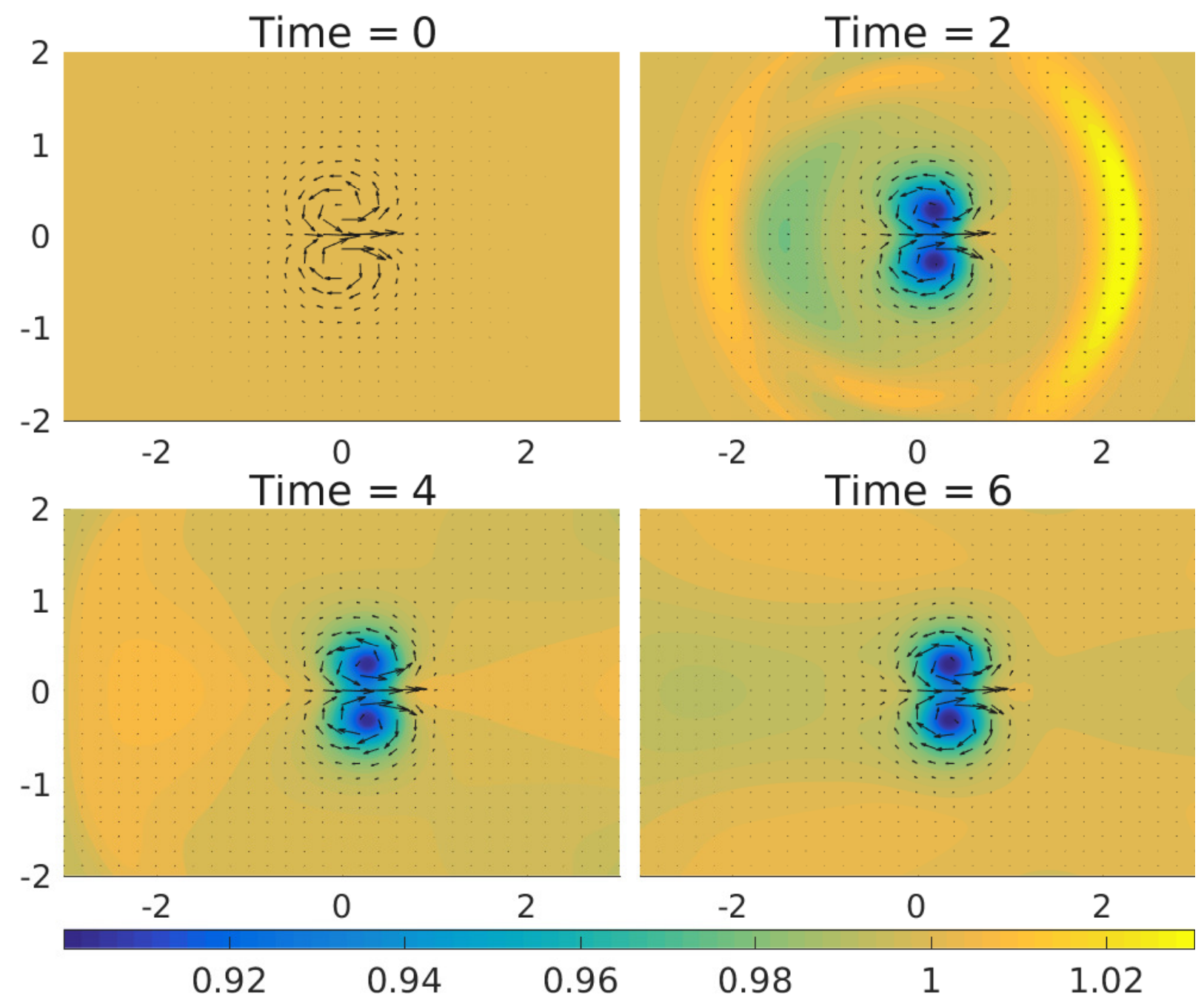

FIG. 2. Initial adjustment of the asymptotic modon with non-dimensional $a=0.5, U=0.15$, as seen in the thickness and velocity fields. Time in units of $1 / \beta L_{d}$.

respectively, which corresponds to nondimensional beta equal to one. Their numerical values, with $g$ and $\beta$ fixed for the Earth, are uniquely defined by the value of $H$, the equivalent depth. In the atmospheric context, for $H \approx 10 \mathrm{~km}, L_{d} \approx 3000 \mathrm{~km}$ and $1 / \beta L_{d} \approx 5 h$. At the initial stages of the simulations, cf. Fig. 2 we observed an adjustment of initial configuration with emission of inertia-gravity waves and formation of a dipolar coherent structure, both in pressure and velocity fields. This dipolar structure in a form of twin cyclones then moves eastward without changing form, as follows from Fig. 3. To exclude the influence of inertiagravity waves produced by the initial adjustment and partially reflected by the boundaries, the modon was "nudged", i.e. the far gravity wave field was removed, and the simulation 


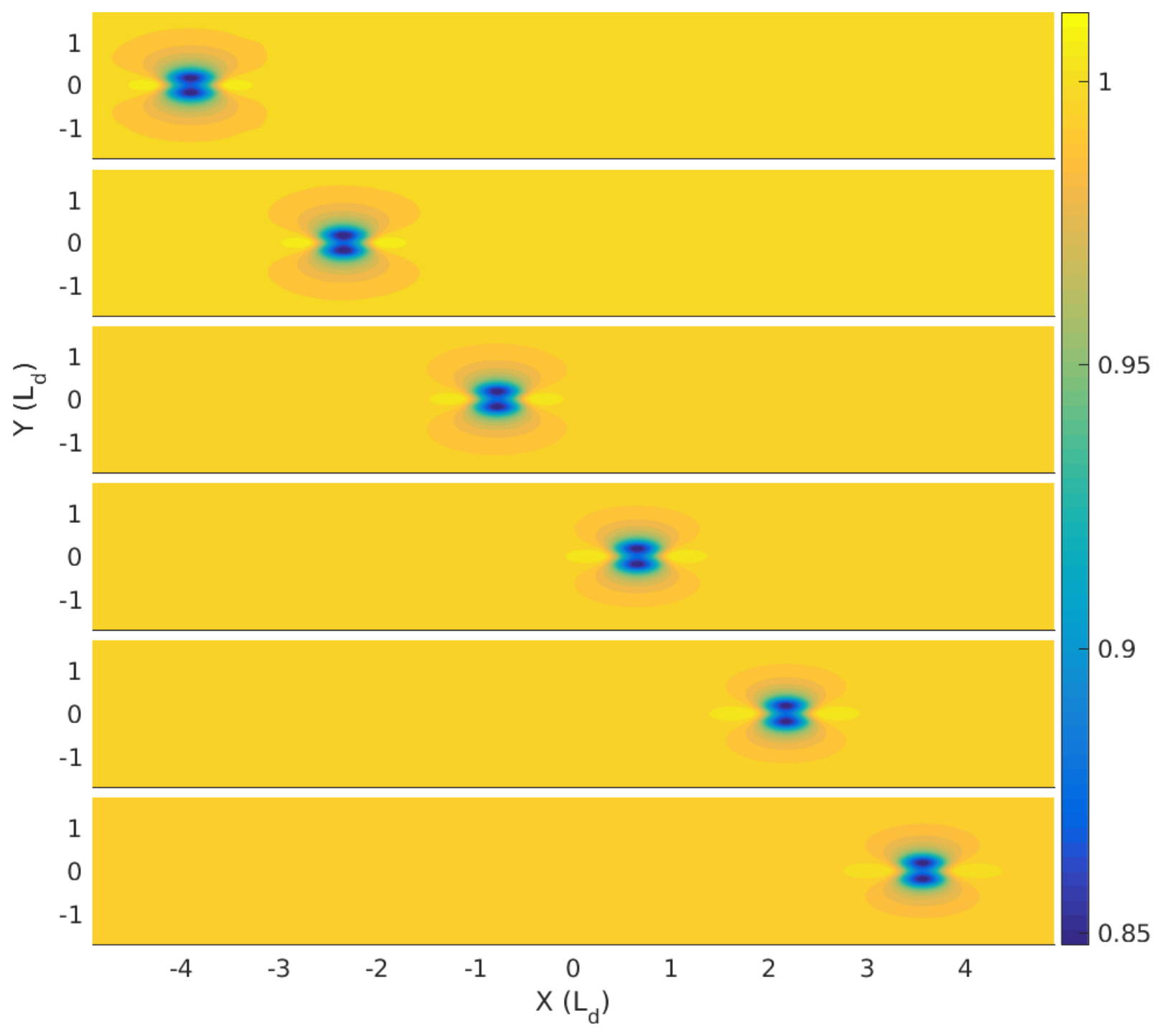

FIG. 3. Snapshots of $h$ for eastward-moving equatorial modon after initial adjustment at $t=$ 0, 10, 20,30,40, $50\left[1 /\left(\beta L_{d}\right)\right]$, from top to bottom (nondimensional $a=0.5, U=0.2$ ).

reinitialized without it. The detailed characteristics of the "exact" modon are presented in Fig. 4. Its coherence can be inferred from the scatter plot of Bernoulli function vs potential vorticity (PV) in the upper-right panel of the Figure, which clearly gives a line. As is known, e.g. [5], Bernoulli function and PV are functionally dependent for steady moving solutions of RSW equations. The phase speed of the modon depends on initial $a, U$. The modons keep moving eastward for the long time without losing their coherence. The modon's velocity can be inferred from the distance it covered with respect to initial condition. The energy of the modon remains practically constant, while we detected a weak enstrophy loss, which 

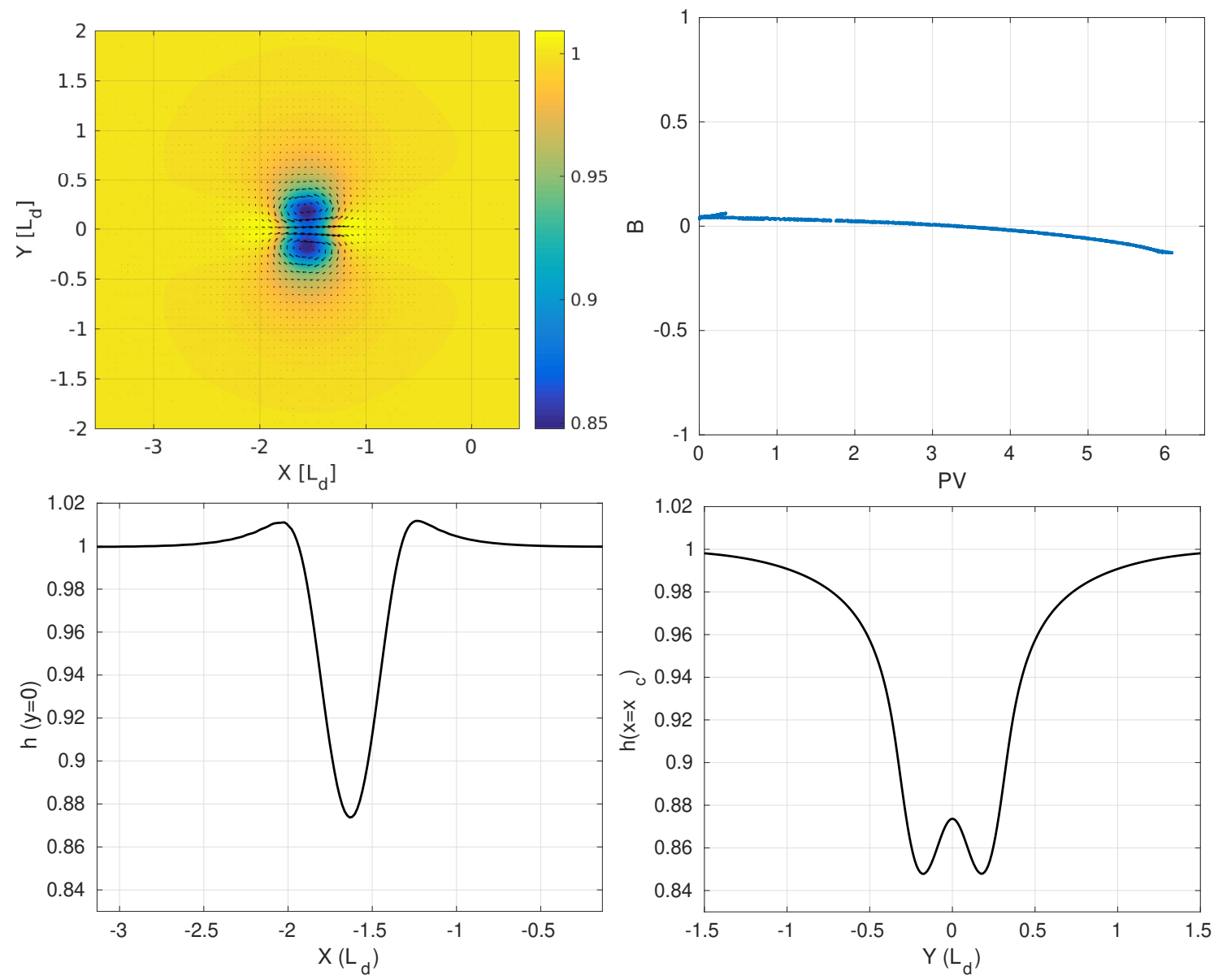

FIG. 4. Upper row: thickness $h$ of the equatorial modon at $t=15\left[1 / \beta L_{d}\right]$ (left); scatter plot of Bernoulli function in the co-moving frame vs potential vorticity (PV) (right). Lower row: Zonal section of the equatorial modon across the center of each cyclone (left), and meridional section across the center of the modon (right). $a=0.5, U=0.2$.

diminishes with increasing resolution (several \% loss at high resolution), not shown. The difference between the asymptotic and "exact" modons is clear from the comparison of their relative vorticity in Figure 5, which shows that the "exact" modon is more compact and has a larger peak vorticity. Although we did not explore in detail the space of parameters of the modon solutions, we varied the parameter $a$, the modon's radius. We observed that while for $a<L_{d}$ equatorial modons always emerge, for $a>L_{d}$, with the same velocity scale, the initial asymptotic modon transforms into a packet of equatorial Rossby waves moving westward. We also checked the robustness of the equatorial modon to the details of initialization, by either removing the outer field of the asymptotic modon, or misaligning its axis. In all 

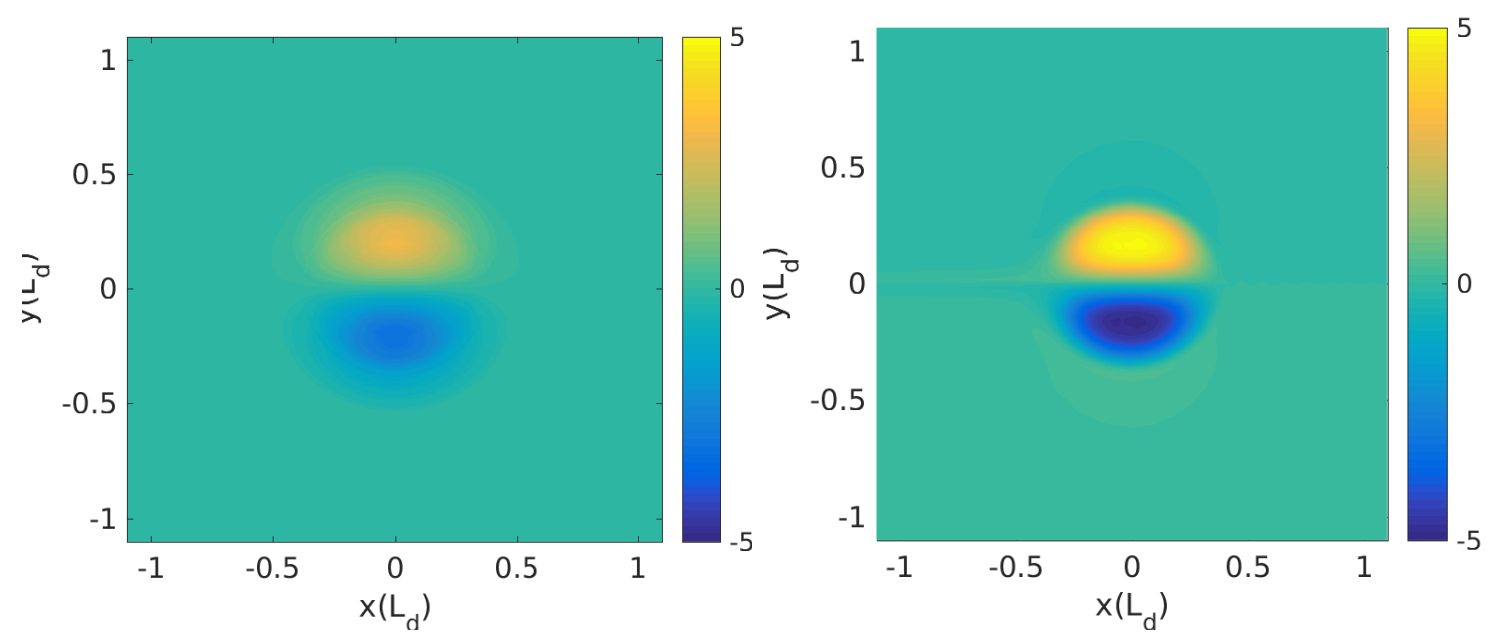

FIG. 5. Relative vorticity of the asymptotic (left) vs "exact" (right) modons. "Exact" modon is more compact and more intense.

such simulations the eastward-moving equatorial modon was always emerging after leaving some "debris" and/or wobbling at initial stages. This indicates that equatorial modons are attracting solutions.

After having established existence of equatorial modons in the adiabatic environment, we switched on the effects of moisture within the diabatic atmospheric moist-convective RSW (mcRSW) [17]. The equations of the model read:

$$
\left\{\begin{array}{l}
\partial_{t} \mathbf{v}+\mathbf{v} \cdot \nabla \mathbf{v}+\beta y \hat{\mathbf{z}} \wedge \mathbf{v}=-g \nabla h \\
\partial_{t} h+\nabla \cdot(\mathbf{v} h)=-\gamma P \\
\partial_{t} Q+\nabla \cdot(Q \mathbf{v})=-P+E
\end{array}\right.
$$

Here $Q$ is a bulk amount of water vapor in the air column, $\gamma$ is a parameter depending on the underlying stratification, $P$ is the condensation sink, and $E$ is the evaporation source of moisture, which are parameterized as follows:

$$
P=\frac{Q-Q^{s}}{\tau} \mathcal{H}\left(Q-Q^{s}\right), \quad E=\alpha|\mathbf{v}|\left(Q^{s}-Q\right) \mathcal{H}\left(Q^{s}-Q\right) .
$$

$Q_{s}$ is a saturation moisture threshold, $\tau$ is relaxation time, $\alpha$ is a parameter regulating evaporation, and $\mathcal{H}(\ldots)$ denotes the Heaviside function. As in previous test simulations [17], we take a uniform initial moisture distribution $Q_{i}$ close to saturation: $Q_{i}=Q_{s}-0.01$ with $Q_{s}=0.9$, a short relaxation time $\tau$ equal to several time-steps of the numerical scheme, $\gamma=1$, and $\alpha=\mathcal{O}\left(10^{-1}\right)$. We performed exactly the same simulations as in the adiabatic 

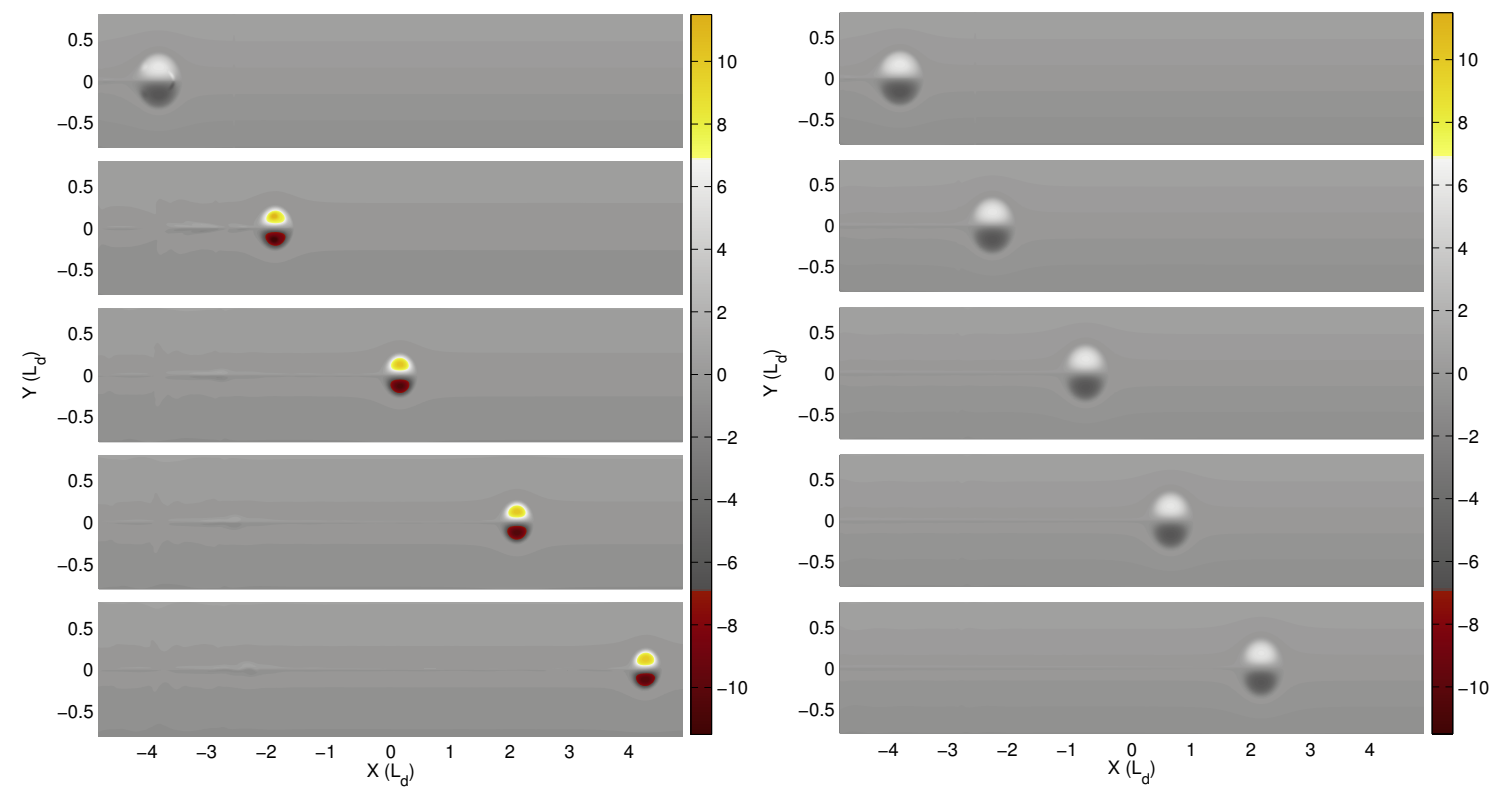

FIG. 6. Steady eastward propagation of the equatorial modon, as seen in PV field in moistconvective (left) and adiabatic (right) environments, with the same initial conditions. $t=$ $0,10,20,30,40\left[1 /\left(\beta L_{d}\right)\right]$, from top to bottom. $(a=0.5, U=0.2, \alpha=0.05)$.

case. They showed that the equatorial modons keep their coherence in the moist-convective environment. Moreover, as is known, and confirmed in mcRSW [19, 20], condensation leads to intensification of the cyclonic vortices. Correspondingly, the potential vorticity (PV) anomalies become stronger, the size smaller, and the phase speed of the convectively-coupled modon becomes higher than that of its adiabatic counterpart. A comparison of evolution of the same modon in adiabatic and moist-convective environments is presented in Fig. 6 . A typical pattern of the associated moisture field, and characteristic condensation patterns at the front and at the rear of the modon are shown in Fig. 7. Such patterns persist all along the simulation. We should stress that, although we initialized the simulations with non-divergent velocity field of the asymptotic modon, the "exact" modon develops and maintains a characteristic convergence/divergence pattern (not shown), which redistributes moisture in its core, and prompts condensation in specific zones. Notice that as condensation enhances cyclonic vorticity [19], the moist-convective modon intensifies, and moves faster than the dry one.

To conclude, we established existence of long-living, slow eastward-moving dipolar coherent structures, the modons, in the shallow-water dynamics in the equatorial beta-plane. In 

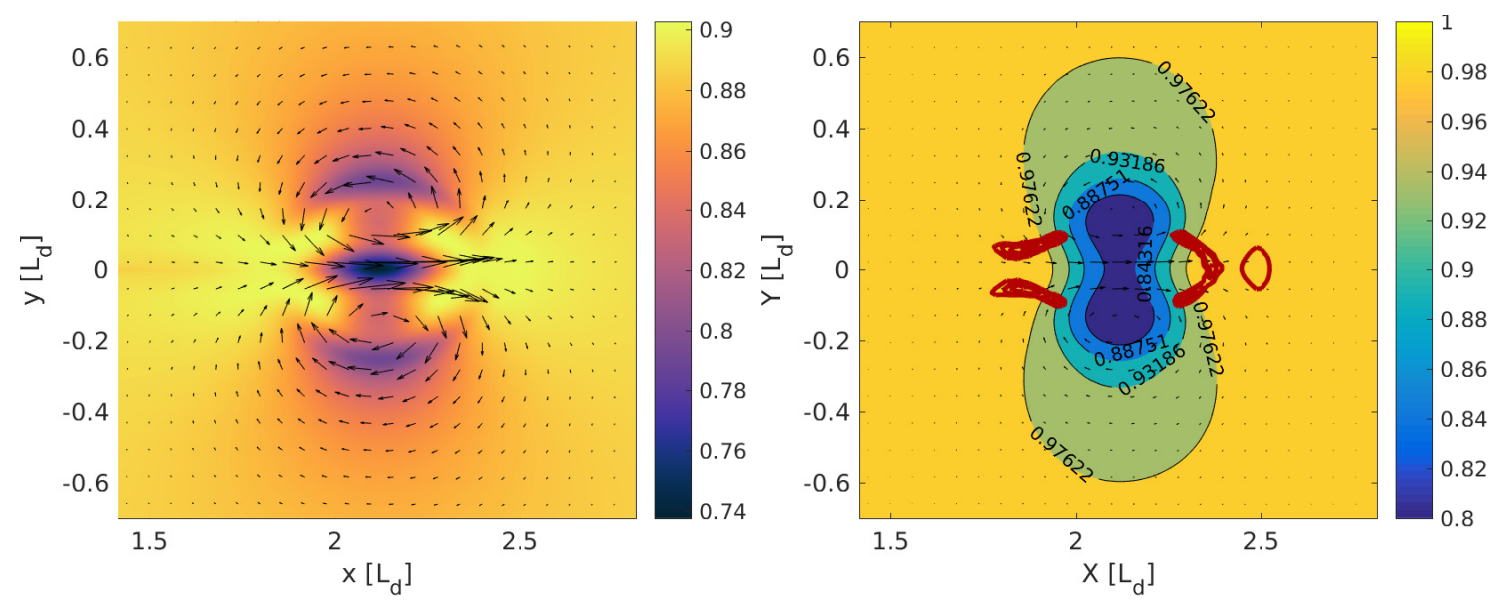

FIG. 7. $(a=0.5, U=0.2, \alpha=0.05)$. Distribution of the water vapor (left) and superposition of pressure (black) and condensation (red) isopleths (right) at $t=30\left[1 /\left(\beta L_{d}\right)\right]$.

the atmospheric context, the modons maintain their coherence in the presence of moisture and are accompanied by a specific and robust moist convection pattern, if condensation and evaporation are present. The scales of these structures are conditioned by the interpretation of the RSW model. At given $g$ and $\beta$ the actual scales depend on the value of the unperturbed thickness $H$, or of the corresponding deformation radius $L_{d}$. Although we cannot make a firm link to MJO at this stage, some features of the equatorial modon do resemble those of dipolar structures associated with the MJO [21]. Work is in progress in this direction, in particular including improved parametrisation of moist processes [20] and vertical structure, which has specific features in MJO, cf. [22] and references therein. Independently of a possible link to MJO, the very existence of the eastward-moving equatorial modons, with their characteristic velocity pattern, slow eastward propagation, and relatively weak signature in pressure should be kept in mind in data analyses and simulations with "big" atmospheric and oceanic models. It is known [23] that long-wave pressure anomalies in RSW at the equator produce westward-propagating dipolar Rossby-wave packets and eastward-propagating Kelvin waves, in accordance with the Gill's scenario [24]. The genesis of eastward-moving dipoles is an important topic, to be addressed elsewhere. Our analysis above suggests that comparable zonal and meridional scales of the initial disturbance, and relative smallness of pressure anomaly are necessary for that.

Let us comment on the relation of equatorial modons we discovered to those known, or 
hypothesized, in the literature. First, while the relation of the spherical modons to the standard QG ones on the midlatitude tangent plane was established in the limit of small spherical modon size [7], this proof, which uses the structural resemblance of equivalent barotropic equations on the sphere and QG equations, both incorporating a finite barotropic deformation radius, is not directly transposable to the equatorial beta-plane case. So the proof that our modons is a limiting case of the spherical ones is pending. Second, westwardpropagating localized solutions, which were also called modons, were constructed in [25] as a generalization of westward-moving equatorial Rossby-wave solitons, and confirmed by direct numerical simulations in [23]. The long-wave scaling used in [25],[23] differs from ours, with the main difference residing in the disparity of zonal and meridional scales. A possibility of existence of eastward-propagating modons, if some ad hoc "heuristic" terms were added to the systematically derived asymptotic equations, was also evoked in [25]. Question remains whether our modons could be related to this conjecture, although the difference in scalings seems to prevent this. We should add, for completeness, that meridionally translating zonally-symmetric modon-like solutions in the vertical plane at the equator were derived in [26] in the context of the atmospheric equatorial boundary layer.

\section{ACKNOWLEDGMENTS}

We are grateful to J.-I. Yano for his constructive remarks and useful discussions. 
[1] M. Wheeler and G. Kiladis, J. Atmos. Sci. 56, 374 (1999).

[2] R. Madden and P. Julian, J. Atmos. Sci. 29, 1109 (1972).

[3] K. Straub and G. N. Kiladis, J. Atmos. Sci. 59, 30 (2002).

[4] V. Larichev and G. Reznik, Doklady USSR Acad. Sci. 231, 1077 (1976).

[5] Z. Kizner, G. Reznik, B. Fridman, R. Khvoles, and J. McWilliams, J. Fluid Mech. 603, 305 (2008).

[6] B. Ribstein, J. Gula, and V. Zeitlin, Phys. Fluids 22, 116603 (2010).

[7] W. Verkley, J. Atmos. Sci. 41, 2492 (1984).

[8] J. Tribbia, Geophys. Astrophys. Fluid Dyn. 30, 131 (1984).

[9] J.-I. Yano and J. Tribbia, J. Atmos. Sci. 74, 3473 (2017).

[10] T. Matsuno, J. Met. Soc. Japan 44, 25 (1966).

[11] I. James, Introduction to Circulating Atmospheres (Cambridge University Press, 1994).

[12] A. Gill, Atmosphere - Ocean Dynamics (Academic Press, 1982).

[13] J. Charney, J. Atmos. Sci. 20, 607 (1963).

[14] J.-I. Yano and M. Bonazzola, J. Atmos. Sci. 66, 159 (2009).

[15] J.-I. Yano, S. Mulet, and M. Bonazzola, Tellus 61A, 417 (2009).

[16] V. Zeitlin, Geophysical Fluid Dynamics: Understanding (almost) everything with rotating shallow water models (Oxford University Press, 2018).

[17] F. Bouchut, J. Lambaerts, G. Lapeyre, and V. Zeitlin, Phys. Fluids 21, 116604 (2009).

[18] F. Bouchut, in Nonlinear Dynamics of Rotating Shallow Water: Methods and Advances, Edited Series on Advances in Nonlinear Science and Complexity, Vol. 2, edited by V. Zeitlin (Elsevier, 2007) pp. $189-256$.

[19] J. Lambaerts, G. Lapeyre, and V. Zeitlin, J. Atmos. Sci. 68, 1234 (2011).

[20] M. Rostami and V. Zeitlin, Q. J. Roy. Met. Soc. 144, 1450 (2018).

[21] H. Rui and B. Wang, J. Atmos. Sci. 47, 357 (1990).

[22] J. Monteiro, A. Adames, W. J.M., and S. J.S., Geoph. res. lett. 41, 9158 (2014).

[23] J. LeSommer, G. Reznik, and V. Zeitlin, J. Fluid Mech. 515, 135 (2004).

[24] A. Gill, Q. J. Roy. Met. Soc. 106, 447 (1980).

[25] J. Boyd, J. Phys. Oceanogr. 15, 46 (1985). 
226 [26] V. Gryanik and A. Dobritsyn, Izvestiya, Atmospheric and Oceanic Physics 26, 260 (1990). 